\title{
A DNA investigation into the mysterious disappearance of the Rocky Mountain grasshopper, mega-pest of the $1800 \mathrm{~s}$
}

\author{
W. Chapco ${ }^{\mathrm{a}, *}$ and G. Litzenberger ${ }^{\mathrm{b}}$ \\ a Department of Biology, University of Regina, 3737 Wascana Parkway, Regina, Sask., Canada S4S 0A2 \\ ${ }^{\mathrm{b}}$ RCMP Forensic Laboratory, Biology Section, 5201 Heather Street, Vancouver, BC, Canada V5Z 3L7
}

Received 24 January 2003; revised 27 May 2003

\begin{abstract}
The mysterious extinction of the Rocky Mountain Grasshopper, Melanoplus spretus, a major pest species on the prairies and plains of the $1800 \mathrm{~s}$, is truly a remarkable event in the history of agriculture. Recently, we obtained specimens of $M$. spretus from museums and from 400-year-old glacial deposits in Wyoming. We report success in obtaining mitochondrial DNA sequences from both sources. This permitted us to examine two issues surrounding the species' disappearance. First, a long-standing view that $M$. spretus and the extant species Melanoplus sanguinipes are (if not phase transforms of one another) sister taxa, is disputed, but cannot be rejected with certainty. Interestingly, there is some evidence that suggests there may be a closer affinity with another member of the Mexicanus species-group, Melanoplus bruneri. Second, because M. spretus still possesses considerable nucleotide diversity $(1.15 \pm 0.19 \%)$, a depletion of variation cannot be considered a factor contributing to its demise.

(C) 2003 Elsevier Inc. All rights reserved.
\end{abstract}

Keywords: Orthoptera; Melanoplinae; mtDNA; Phylogenetics

\section{Introduction}

Exactly 100 years ago, the last recorded living specimen of the Rocky Mountain Grasshopper, Melanoplus spretus, was collected in Aweme, near Brandon, Manitoba (Gurney and Brooks, 1959). The major pest of the 19th century prairies, M. spretus attained immeasurable population sizes from 1850 to 1880 before crashing soon after. Subsequently, the species never recovered. Its disappearance possibly "represents the only extinction of a pest species in the history of agriculture" (Lockwood and DeBrey, 1990). Several extinction theories have been advanced, including one in which the species' demise is attributed to the disappearance of the great buffalo herds coupled with large decreases in Indian populations. The widespread introduction in the 1880s of alfalfa, known for its harmful effects on Melanoplus, has also been considered

\footnotetext{
${ }^{*}$ Corresponding author. Fax: +1-306-337-2410.

E-mail addresses: Chapco@uregina.ca (W. Chapco), Greg.Litzenberger@rcmp-grc.gc.ca (G. Litzenberger).
}

as a contributing factor (Brett, 1947; Gurney and Brooks, 1959). Addressing these and other speculations, Lockwood and DeBrey (1990) discuss and refute most of them. Ultimately, they ascribe the species' extinction to the destruction of oviposition and development sites, particularly in riparian areas (their preferred habitats), brought about by human expansion and agriculture, all coincident with a natural population decline. Conversely, Otte (2002) suspects that M. spretus may not be extinct but, instead, lies dormant awaiting conditions suitable for rejuvenation. While cases of prolonged persistence in refugia during non-outbreak years are known for some insect pest species (Wallner, 1987), we are not aware of any whose duration is as long as 100 years. Others believe that M. spretus was a migratory phase of the extant Melanoplus sanguinipes, and that today is in fact present as the latter species (Capinera and Sechrist, 1982). This viewpoint has subsequently led to some heated debate concerning the species status of $M$. spretus and the species-defining value of genitalic characters (Cohn, 1994; Lockwood, 1996). Not considered in these scenarios, or indeed in most accounts of extinction involving wildlife populations 
(Frankham, 1995), is the possibility that such disappearances stemmed from interactions between genetic and non-genetic elements. Given the albeit irregular cyclic nature of population density fluctuations in many pest species of Melanoplus (Riegert, 1968), it is distinctly possible that in $M$. spretus' distant past, prior to the population explosions of the mid-1800s, the species had experienced repeated bottlenecks, leaving it bereft of variation (see Motro and Thomson, 1982 for theoretical discussions) and poorly adapted to deal with subsequent onslaughts of human activity.

Recently, we received from colleagues eight museum $M$. spretus specimens and six ca. 400-year-old, intact and partial specimens from Knife Point Glacier, Wyoming, identified as $M$. spretus morphometrically (Lockwood et al., 1994; Lockwood, 2002) and by comparative cuticular hydrocarbon profiles (Sutton et al., 1996). Our objectives were to determine whether DNA could be isolated from these remains, sequenced, and used to evaluate $M$. spretus' (a) connection with $M$. sanguinipes and (b) diversity level compared to its congeners.

\section{Materials and methods}

Information on all museum and glacial specimens of M. spretus is set out in Table 1. To ascertain M. spretus' phylogenetic position within the genus, these individuals and 38 specimens representing 19 divergent species of Melanoplus (Chapco and Litzenberger, 2002) were analyzed. Included were, respectively, two and three specimens of $M$. mexicanus and Melanoplus bruneri (Table 1), not heretofore sequenced. Five species (borealis, bruneri, devastator, sanguinipes, and mexicanus) belong to species-group Mexicanus, of which $M$. spretus is also considered a member (Gurney and Brooks, 1959). The analysis incorporated all three recognized subspecies of M. sanguinipes (Gurney and Brooks, 1959). Schistocerca gregaria and Locusta migratoria served as outgroup taxa.

The "CTAB/DTAB" method of Phillips and Simon (1995) proved successful in extracting DNA from all museum and glacial specimens. Regions of four mitochondrial genes (cytb, COII, ND2, and COI) were amplified and sequenced, following procedures described in Litzenberger and Chapco (2001a,b). Primer sequences are described in Litzenberger and Chapco (2001a,b). For older specimens, including those of M. spretus, amplification could only be achieved using primers spanning shorter, overlapping, portions of each region. [For M. spretus, primers CO2E and mtD11, cited in the above references, were replaced by new primers:

CO2F 5'-GACCAAAAAATAATCCTGGTCGG- $3^{\prime}$ and

CO1G 5'-AGCTCATACAATAAATCCTATTAGT C- $3^{\prime}$.

Positions of the $5^{\prime}$ nucleotides relative to Locusta are 3623 and 2293, respectively.]

Resultant sequences were easily aligned by visual inspection and imported into MacClade (Maddison and Maddison, 1992). Phylogenetic relationships were inferred using maximum parsimony (MP) and weighted parsimony (wMP), following Farris' (1969) iterative reweighting scheme, both available in the software package, PAUP, version 4.0b8 (Swofford, 2001). For all analyses, the four sequences were treated as a combined unit, a procedure that, as in all our previous studies

Table 1

Specimens, sources, and accession numbers

\begin{tabular}{|c|c|c|c|}
\hline Specimen/sex & Donor & Source, year of collection & Accession Nos. (cytb, COII, ND2, COI) \\
\hline M. spretus $1 /$ + & Otte & Argentine Pass, CO, 1877 & AF372990-AF372993 \\
\hline M. spretus $2 /$ ? & Otte & Barras, Mexico, year? & AY147116-AY 147119 \\
\hline M. spretus $3 / \widehat{\jmath}$ & Kondratieff & Larimore, ND, 1891 & AY147120-AY147123 \\
\hline 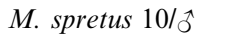 & Lockwood & Turtle Mt., ND, 1877 & AY147148, AY147149, -, - \\
\hline M. spretus $11 /$ ? & Otte & Salt Lake City, UT, 1877 & $-, \mathrm{AY} 147150,-,-$ \\
\hline M. spretus $4 /$ ? & Lockwood & Knife Point Glacier, WY & AY147124-AY147127 \\
\hline M. spretus $5 /$ ? & Lockwood & Knife Point Glacier, WY & AY147128-AY147131 \\
\hline M. spretus $6 /$ ? & Lockwood & Knife Point Glacier, WY & AY147132-AF147135 \\
\hline M. spretus $7 /$ ? & Lockwood & Knife Point Glacier, WY & AY147136-AF147139 \\
\hline M. spretus $8 / ?$ & Lockwood & Knife Point Glacier, WY & AY147140-AF147143 \\
\hline M. spretus $9 / 9$ & Lockwood & Knife Point Glacier, WY & AY147144-AF147147 \\
\hline M. spretus/q & Otte & Manitoba, year? & No DNA \\
\hline M. spretus/ð & Otte & Colorado, year? & No DNA \\
\hline M. spretus/? & Boucher & Manitoba, 1901 & No DNA \\
\hline M. mexicanus $1 / \widehat{\jmath}$ & Otte & Mexico, 1936 & AY147151-AY147154 \\
\hline 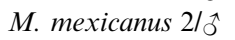 & Otte & Mexico, 1936 & AY147155-AY147158 \\
\hline M. bruneri $2 / \widehat{0}$ & Chapco & Saskatchewan, 1999 & AY303817-AY303820 \\
\hline M. bruneri $3 / \widehat{0}$ & Chapco & Saskatchewan, 1999 & AY303821-AY303824 \\
\hline M. bruneri $4 / \widehat{o}$ & Chapco & Saskatchewan,1999 & AY303825-AY303828 \\
\hline
\end{tabular}


(Chapco et al., 2001; Litzenberger and Chapco, 2001a,b), always yielded trees with greater resolution and bootstrap support when compared to those based on single genes. Levels of support for derived relationships were estimated through 1000 bootstrap replicates. Within species nucleotide diversities were estimated using MEGA version 2.1 (Kumar et al., 2001).

\section{Results and discussion}

Sequences were successfully obtained from five museum specimens and from all six individuals recovered from the glacier. Three museum specimens yielded no DNA, and in two cases (\#10 and \#11) only one or two genes could be sequenced (Table 1). Sequence data

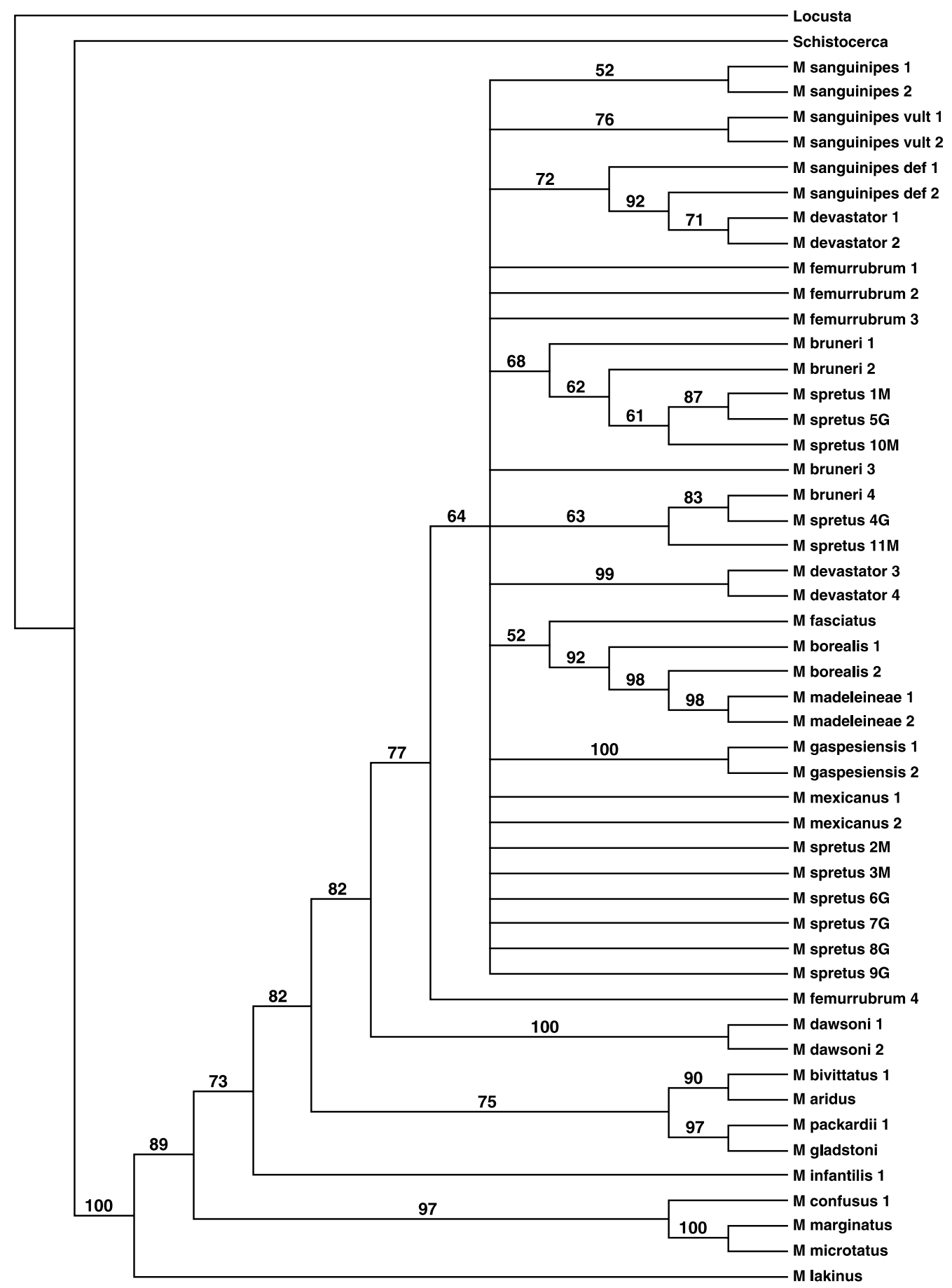

Fig. 1. Bootstrap (1000 replicates) weighted maximum parsimony tree. Bootstrap values $\geqslant 50 \%$ are indicated. $M$ and $G-$ museum and glacial specimens. 
(a maximum of 1716 bases) have been deposited in GenBank. Across the four genes, 588 sites were variable and of these, 297 were phylogenetically informative. Maximum parsimony yielded $>40,000$ equally parsimonious trees, each of length 883 steps and consistency index $43.9 \%$. Weighted parsimony recovered 450 trees of length 198.5 and consistency index $65.5 \%$. Relationships were essentially the same as those recovered by MP, but resolution was better using wMP (Fig. 1). All spretus individuals fell ( $82 \%$ bootstrap support) well within the large clade (sanguinipes...dawsoni), labeled " $\mathrm{A}$ " in Chapco and Litzenberger (2002), which not only included all members of the Mexicanus species-group but five other species, some previously assigned to other species-groups, some unassigned. The degree of resolution within the clade is variable. Like for most species in the clade, specimens of M. spretus do not emerge as a monophyletic assemblage, but instead, where there is bootstrap support, they are intertwined with bruneri and not sanguinipes individuals [Such patterns have been attributed to incomplete "lineage sorting" of alleles derived from a recent, common, polymorphic ancestor (Chapco and Litzenberger, 2002; Knowles and Otte, 2000).] There is some statistical support for this unexpected connection between $M$. spretus and M. bruneri. Kishino and Hasegawa (1989) log-likelihood ratio tests were applied to two tree topologies, emerging from additional wMP searches in which clustering between spretus and each of bruneri and sanguinipes was imposed, and compared to "the" wMP tree. [Since all searches, constrained or unconstrained, yielded multiple wMP trees, single trees each with the numerically largest log-likelihood were used in the tests.] The unconstrained wMP tree had the largest $\log$-likelihood $(-\ln L=$ 9105.89), a value significantly greater than that of the constrained tree connecting spretus and sanguinipes specimens $(\Delta \ln L=153.76 \pm 51.10 ; P \approx 0.003)$. In contrast, the wMP tree was not significantly different from one in which monophyly was imposed on spretus and bruneri individuals $(\Delta \ln L=47.81 \pm 37.96 ; P \approx 0.21)$. One interpretation, therefore, is that during the relatively rapid sequence of radiation events, now believed to characterize the genus Melanoplus (Chapco and Litzenberger, 2002; Knowles and Otte, 2000), a cluster of spretus and bruneri "alleles" had segregated from the common polymorphic ancestor of group "A." However, without concordant support from other gene genealogies, nuclear-based ones in particular, extrapolating to the species level (Avise, 1994) and concluding that spretus' nearest relative is not sanguinipes but bruneri may be premature.

Nucleotide diversities of museum, glacial, and combined specimens are, respectively, $1.18 \pm 0.23 \%$, $1.06 \pm 0.20 \%$, and $1.15 \pm 0.19 \%$, all well within the range of published values for Melanoplus species (Chapco and Litzenberger, 2002). Admittedly, we did not sample in- sects at, or near, their time of extinction, a nearly impossible feat given the rarity of available specimens and our dependence on the generosity of others. In any event, diversity would have been expected to have been low even during $M$. spretus' great rise in numbers if it had indeed experienced a series of genetic bottlenecks in the past. Although clearly this is not the case, it remains obvious that $M$. spretus lacked the specific genetic machinery, unlike its congeners, to cope with environmental changes wrought by the early settlers (Lockwood and DeBrey, 1990). On a final note, where there is good resolution, glacial and museum specimens pair up rather well, a result that should be gratifying to those who have had to rely on non-molecular methods for identification.

\section{Acknowledgments}

We are very grateful to S. Boucher (Lyman Entomological Museum, McGill University), B. Kondratieff (Colorado State University), and D. Otte (The Academy of Natural Sciences of Philadelphia) for their generous providing of museum specimens of Melanoplus spretus. We particularly thank J. Lockwood for his kind donation of both museum and glacial materials. This research was funded by a grant (W.C.) and PGSB scholarship (G.L.) from the Natural Sciences and Engineering Research Council of Canada.

\section{References}

Avise, J.C., 1994. Molecular Markers, Natural History and Evolution. Chapman and Hall, NY.

Brett, C.H., 1947. Interrelated effects of food, temperature, and humidity on the development of the lesser migratory grasshopper, Melanoplus mexicanus mexicanus (Saussure) (Orthoptera). Tech. Bull. Okla. Agr. Exp. Sta. T-26, 50.

Capinera, J.L., Sechrist, T.S., 1982. Grasshoppers (Acrididae) of Colorado: identification, biology and management. Colo. State Univ. Exp. Stn. Bull., 584S.

Chapco, W., Litzenberger, G., 2002. A molecular phylogenetic analysis of the grasshopper genus Melanoplus (Stål) (Orthoptera: Acrididae) - an update. J. Orthopt. Res. 11, 1-9.

Chapco, W., Litzenberger, G., Kuperus, W.R., 2001. A molecular biogeographic analysis of the relationship between North American melanopline grasshoppers and their Eurasian and South American relatives. Mol. Phylogenet. Evol. 18, 460-466.

Cohn, T.J., 1994. The use of male genitalia in taxonomy and comments on Lockwood's 1989 paper on Melanoplus spretus (Walsh). J. Orthopt. Res. 3, 59-63.

Farris, J.S., 1969. A successive approximations approach to character weighting. Syst. Zool. 18, 374-385.

Frankham, R., 1995. Conservation genetics. Annu. Rev. Genet. 29, 305-327.

Gurney, A.B., Brooks, A.R., 1959. Grasshoppers of the mexicanus group, genus Melanoplus (Orthoptera: Acrididae). Proc. US Natl. Mus. 110, 1-93.

Kishino, H., Hasegawa, M., 1989. Evaluation of the maximum likelihood estimate of the evolutionary tree topologies from 
DNA sequence data, and the branching order in hominoidea. J. Mol. Evol. 29, 170-179.

Knowles, L.L., Otte, D., 2000. Phylogenetic analysis of montane grasshoppers from western North America (Genus Melanoplus, Acrididae: Melanoplinae). Ann. Entomol. Soc. Am. 93, 421431.

Kumar, S., Tamura, K., Jakobsen, I.B., Nei, M., 2001. MEGA2: Molecular Evolutionary Genetics Analysis software. Arizona State University, Tempe, Arizona, USA.

Motro, U., Thomson, G., 1982. On heterozygosity and the effective size of populations subject to size changes. Evolution 36, 10591066.

Litzenberger, G., Chapco, W., 2001a. A molecular phylogeographic perspective on a fifty-year-old taxonomic issue in grasshopper systematics. Heredity 86, 54-59.

Litzenberger, G., Chapco, W., 2001b. Molecular phylogeny of selected Eurasian podismine grasshoppers (Orthoptera: Acrididae). Ann. Entomol. Soc. Am. 94, 505-511.

Lockwood, J.A., 2002. pers. comm.

Lockwood, J.A., DeBrey, L.D., 1990. A solution for the sudden and unexplained extinction of the Rocky Mountain Grasshopper (Orthoptera: Acrididae). Environ. Entomol. 19, 1194-1205.

Lockwood, J.A., DeBrey, L.D., Thompson, C.D., Love, C.M., Nunamaker, R.A., Shaw, S.R., Schell, S.P., Bomar, C.R., 1994. Preserved insect fauna of glaciers of Fremont County in Wyoming: insights into the ecology of the extinct Rocky Mountain Locust. Environ. Entomol. 23, 220-235.

Lockwood, J.L., 1996. Phallic fact, fallacies, and fantasies: comments on Cohn's 1994 paper on Melanoplus spretus (Walsh). J. Orthopt. Res. 5, 57-66.

Maddison, W.P., Maddison, D.R., 1992. MacClade: analysis of phylogeny and character evolution, version 3.01. Sinauer, Sunderland, MA.

Otte, D., 2002. In New York Times. April 23 issue.

Phillips, A.J., Simon, C., 1995. Simple, efficient, and nondestructive DNA extraction protocol for arthropods. Ann. Entomol. Soc. Am. 88, 281-283.

Riegert, P.W., 1968. A history of grasshopper abundance surveys and forecasts of outbreaks in Saskatchewan. Mem. Entomol. Soc. Can. (52), 99.

Sutton, B.D., Carlson, D.A., Lockwood, J.A., Nunamaker, R.A., 1996. Cuticular hydrocarbons of glacially-preserved Melanoplus (Orthoptera: Acrididae): identification and comparison with hydrocarbons of M. sanguinipes and M. spretus. J. Orthopt. Res. 5, 1-12.

Swofford, D.L., 2001. PAUP*. Phylogenetic Analysis Using Parsimony (*and Other Methods). Version 4.0b8. Sinauer, Sunderland, MA.

Wallner, W.E., 1987. Factors affecting insect population dynamics: differences between outbreak and non-outbreak species. Annu. Rev. Entomol. 32, 317-340. 